\title{
Performance Analysis of Two-Hop Amplify and Forward Relayed Transmission in Asymmetric Rayleigh and Rician Fading Environment
}

\author{
Priyanka Mehta \\ Department of Electronics and \\ Communication Engineering \\ FET, Mody Institute of \\ Technology and Science \\ (Deemed University) \\ Lakshmangarh, Dist. Sikar, \\ Rajasthan 332311, India
}

\author{
Suman Rathore \\ Department of Electronics and \\ Communication Engineering \\ FET, Mody Institute of \\ Technology and Science \\ (Deemed University) \\ Lakshmangarh, Dist. Sikar, \\ Rajasthan 332311, India
}

\author{
Kapil Gupta \\ Department of Electronics and \\ Communication Engineering \\ FET, Mody Institute of \\ Technology and Science \\ (Deemed University) \\ Lakshmangarh, Dist. Sikar, \\ Rajasthan 332311, India
}

\begin{abstract}
This paper present the performance analysis of two-hop relayed transmission over asymmetric Rayleigh and Rician fading environment. In many scenarios the commonly assumed symmetry in relay channels is unrealistic. Therefore this paper used asymmetric fading condition where the link experience unequal signal strength. Closed form expression for Outage Probability and Average Bit Error Probability are derived to study the performance of the system and also illustrate the positive impact of the Rician parameter on the system performance.
\end{abstract}

\section{Keywords}

Relay; Amplify-and-forward; Channel State Information(CSI) assisted relay; Rayleigh fading; Rician fading; Line of sight(LOS); Non line of sight(NLOS); Outage Probability; Average Bit Error Probability (ABEP).

\section{INTRODUCTION}

Relaying technique is used for extending the coverage area (possibly shadowed) in wireless environments. Consider a communication system in which two terminals are communicating via a third terminal that acts as a relay. Relay is the repeater, node or channel that inter connects the source (transmitter) and destination (receiver), through which they can receive and transmit information from source to destination, when the direct link between the source and destination is in deep fade, so the signals to the destination propagate through two or more hops or links in series [1]. Relay is used to boost and resend a signal to the next terminal. Relay transmission has been identified as one of the core technologies that could enable high speed information transfer over challenging wireless environment [2]. Relay has made a major part in mobile communication and wireless broadband access. This scenario was encountered originally in bent-pipe satellites where the primary function of the spacecraft is to relay the uplink carrier into a downlink [3]. It is also common in various fixed microwave links by enabling greater coverage without the need of large power at the transmitter. More recently, this concept has gained new actuality in collaborative/cooperative wireless communication systems. Few statistical models are used to describe fading in wireless environments in communication system analysis. The most frequently used distributions are Rayleigh, Rician, Nakagami$\mathrm{m}$, Nakagami-q and Wiebull.
Two-hop transmission system can be classified into two main categories, depending on the nature and complexity of the relays, 1) Regenerative relay systems and 2) Non-regenerative relay systems. In the case of regenerative also known as decode-and-forward systems, the relay fully decodes the signal that went through the first hop and retransmits the decoded version into the second hop. Non regenerative also known as amplify-and-forward systems use less complex relays that just amplify and forward the incoming signal without performing any sort of decoding.

Relay in non-regenerative systems can in their turn be classified into two sub categories blind relay and channel state information (CSI) assisted relay. In reference paper [4], systems with 'blind' relay employ at the relay amplifiers with fixed gain and consequently result in a signal with variable power at the relay output. On the other hand, non-regenerative systems with CSI-assisted relay use instantaneous CSI if the first hop to control the gain introduce by the relay and as result fix the power of the retransmitted signal.

We can divide the prior related research into two main categories. The first category deals with the performance analysis over the symmetric fading channels for the relay networks (two-way, dual-hop, multi-hop, and multi-cast) [3, 5]. The second category deals with the performance of relay networks over asymmetric fading channels [6, 7]. In asymmetric fading each link in relay network may have different fading channel.

In this paper we have considered source-relay and relaydestination channel that experience Rayleigh and Rician fading respectively. Rician fading is used for Line of sight path and Rayleigh fading is used where there are many objects in the environment that scatter the radio signal and create multipath fading, and also closed form expression for statistics of received signal to noise ratio (SNR) is analyzed. We basically analyze results with the help of PDF and CDF of destination SNR and then derive various performance measures to study the positive impact of the Rician factor on the system performance. It is also shown that best performance may be achieved when fading parameter $(\mathrm{K})$ is increasing. We derived exact expression of average bit error probability for QPSK and BPSK modulation schemes and also outage probability is derived in order to investigate effect of fading factor. 
The organization of the paper is as follows. Section 2 describes the system and channel model. The expressions for the outage probability and average bit error probability are derived in section 3. Section 4 discusses the numerical results. Finally, conclusion is given in section 5 .

\section{SYSTEM AND CHANNEL MODEL}

We consider a two hop transmission system shown in figure 1 where the communication from the source $\mathrm{S}$ to the destination $D$ via a relay $R$ takes place in two time slots. In the first time slot, $\mathrm{S}$ is transmitting a signal $\mathrm{s}(\mathrm{t})$ to $\mathrm{R}$ which has an average power of $P_{1}$. The received signal at terminal $\mathrm{R}$ can be written as,

$R_{r}(t)=h_{S R} s(t)+n_{1}(t)$

where $h_{S R}$ is the fading amplitude of the channel between $\mathrm{S}$ and $\mathrm{R}$, and $n_{1}(t)$ is an additive white Gaussian noise (AWGN) signal with a power of $N_{0}$.

In the second time slot, $\mathrm{R}$ amplifies the received signal by a gain factor $\mathrm{G}$ and forwards the resultant signal to $\mathrm{D}$ which has an average power of $P_{2}$. The received signal at terminal D can be written as,

$R_{D}(t)=h_{R D} G\left(h_{S R} s(t)+n_{1}(t)\right)+n_{2}(t)$

where $h_{R D}$ is the fading amplitude of the channel between terminal $\mathrm{R}$ and $\mathrm{D}$, and $n_{2}(t)$ is an additive white Gaussian noise (AWGN) signal with a power of $N_{0}$.

The instantaneous end-to-end SNR $\gamma_{e q}$ at the destination can be written as [8],

$\gamma_{e q}=\frac{\left(\frac{P_{1}}{N_{0}}\right)\left|h_{S R}\right|^{2}\left(\frac{P_{2}}{N_{0}}\right)\left|h_{R D}\right|^{2}}{\left(\frac{P_{2}}{N_{0}}\right)\left|h_{R D}\right|^{2}+\left(\frac{1}{G^{2} N_{0}}\right)}$

It is clear from equation (3) that the choice of the relay gain defines the equivalent end-to-end SNR of the two hops. If G is selected according to the instantaneous CSI assisted relay gain [3], then $\gamma_{e q}$ can be re-expressed as

$\gamma_{e q}=\frac{\gamma_{1} \gamma_{2}}{\gamma_{1}+\gamma_{2}+c}$

where $\gamma_{1}=\left|h_{S R}\right|^{2}\left(\frac{P_{1}}{N_{0}}\right)$ and $\gamma_{2}=\left|h_{R D}\right|^{2}\left(\frac{P_{2}}{N_{0}}\right)$ are the per hop SNRs. In addition, exact $\gamma_{e q}$ is given by substituting $\mathrm{c}=1$, and well approximated at high SNR by substituting $\mathrm{c}=0$.

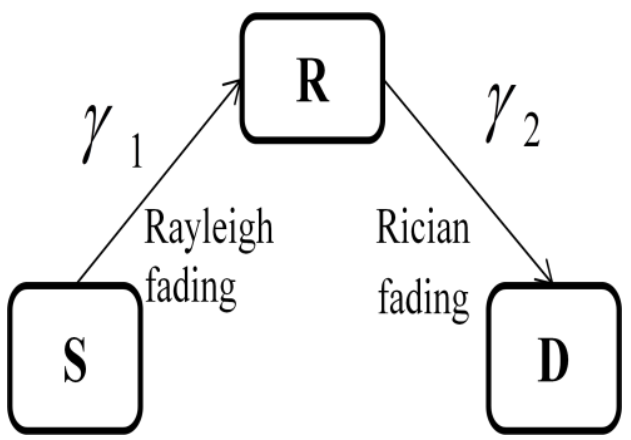

Fig.1: Asymmetric fading channel model

Consider an asymmetric scenario for the fading distributions of the $S \rightarrow R$ and $R \rightarrow D$ links, namely The $S \rightarrow R$ link is subject to Rayleigh fading and the $\mathrm{R} \rightarrow \mathrm{D}$ link is subject to Rician fading. The proposed model in [3] represent either an up or down link in a mobile communication network. In the first case a mobile station acts as $\mathrm{S}$, another mobile station as $\mathrm{R}$ and a base station as D. Note that due to the symmetry of $\gamma_{\mathrm{eq}}$ w.r.t to $\gamma_{1}$ and $\gamma_{2}$, the statistics of $\gamma_{\mathrm{eq}}$ will be identical in the down link where the $\mathrm{S}-\mathrm{R}$ link is subject to Rayleigh fading and the $\mathrm{R}-\mathrm{D}$ link is subject to Rician fading.

In first time slot if the $\mathrm{S}$ is working like a mobile station and the relay work as mobile link, mobile station and mobile link having multiple path so source relay link experiences Rayleigh fading, $\quad \gamma_{1}$ is exponentially distributed with probability density function (PDF) given by [9],

$p_{\gamma_{1}}(\gamma)=\frac{1}{\bar{\gamma}_{1}} \exp \left(-\frac{\gamma}{\bar{\gamma}_{1}}\right)$

where $\overline{\gamma_{1}}=\mathrm{E}\left\{\left|\mathrm{h}_{\mathrm{SR}}\right|^{2}\left(\frac{\mathrm{P}_{1}}{\mathrm{~N}_{0}}\right)\right\}$ is the average per hop SNR of the $\mathrm{S} \rightarrow \mathrm{R}$ channel and $\mathrm{E}\{$.\} is the statistical expectation.

Now in second time slot Destination work as a Base Station and Relay Station work as mobile link, so relay destination link experiences Rician fading It is used to model a propagation path that consists of one strong direct Line of sight (LOS) component and many other weaker non Line of sight (LOS) components. Rician fading parameter $\mathrm{K}$ is measure of severity of fading and SNR of second hop $\gamma_{2}$ is distributed according to a noncentral- $\chi 2$ distribution given by

$p_{\gamma_{2}}(\gamma)=\frac{(K+1)}{\overline{\gamma_{2}}} \exp \left(-K-\frac{(K+1) \gamma}{\overline{\gamma_{2}}}\right) I_{0}\left(2 \sqrt{\frac{K(K+1) \gamma}{\overline{\gamma_{2}}}}\right)$

where $K$ is the Rician factor defined as the ratio of the powers of the LoS component to the scattered components, and $\bar{\gamma}_{2}=E\left\{\left|h_{R D}\right|^{2}\left(\frac{P_{2}}{N_{0}}\right)\right\}$ is the average per hop SNR of the $\mathrm{R} \rightarrow \mathrm{D}$ channel and $I_{0}($.$) is the zeroth order modified Bessel function$ of the first kind [10]. We include Rayleigh fading (when $\mathrm{K}=$ 0 ) and AWGN (when $K=\infty$ ), as special case of no fading.

\section{PERFORMANCE ANALYSIS}

\subsection{Average Signal to Noise Ratio}

Probably the most common and well understood performance measure characteristic of a digital communication system is signal-to-noise ratio (SNR). Most often this is measured at the output of the receiver [9], and is thus directly related to the data detection process itself. the several possible performance measures that exist, it is typically the easiest to evaluate and most often serves as an excellent indicator of the overall fidelity of the system. While traditionally the term "noise" in signal to noise ratio refers to the ever-present thermal noise at the input to the receiver, in the context of a communication system subject to fading impairment, the more appropriate performance measure is average SNR, where the term "average" refers to statistical averaging over the probability distribution of the fading. In simple mathematical terms, if $\gamma$ denotes the instantaneous SNR at the receiver output that includes the effect of fading, then

$\bar{\gamma}=\int_{0}^{\infty} \gamma p_{\gamma}(\gamma) d \gamma$

is the average $\mathrm{SNR}$, where $p_{\gamma}(\gamma)$ denotes the probability density function (PDF) of $\gamma$.

\subsection{Outage Probability}

Outage Probability is an important performance measure that is commonly used to characterize a wireless communication system. It is defined as the probability that the instantaneous 
end-to-end SNR falls below a predefined threshold $\gamma_{t h}$ [9]. Therefore mathematically, the outage probability is

$P_{\text {out }}=F_{\gamma_{\text {eq }}}\left(\gamma_{\text {th }}\right)=P_{r}\left[\frac{\gamma_{1} \gamma_{2}}{\gamma_{1}+\gamma_{2}+c}<\gamma_{\text {th }}\right]$

where $F_{\gamma_{e q}}\left(\gamma_{t h}\right)$ is the CDF of the end-to-end SNR. After applying some algebraic manipulations $F_{\gamma_{e q}}\left(\gamma_{t h}\right)$ can be reexpressed

$F_{\gamma_{e q}}\left(\gamma_{t h}\right)=1-\int_{0}^{\infty} C_{\gamma_{1}}\left(\gamma_{t h}+\frac{\gamma_{t h}{ }^{2}+c \gamma_{t h}}{w}\right) p_{\gamma_{2}}\left(\gamma_{t h}+w\right) d w$

where $\mathrm{C}_{\gamma_{\mathrm{i}}}()=.1-\mathrm{F}_{\gamma_{\mathrm{i}}}($.$) is the complementary CDF of \gamma_{i}$. We obtained $\mathrm{F}_{\gamma_{\text {eq }}}\left(\gamma_{\text {th }}\right)$ by substituting equation (5) and (6) in (9), which can be written as,

$\mathrm{F}_{\gamma_{\mathrm{eq}}}\left(\gamma_{\mathrm{th}}\right)=1-\frac{(K+1)}{\overline{\gamma_{2}}} e^{-K-\left(\frac{1}{\bar{\gamma}_{1}}+\frac{(K+1)}{\overline{\gamma_{2}}}\right) \gamma_{\text {th }}} \times$

$\int_{0}^{\infty} \mathrm{e}^{-\frac{\gamma_{\mathrm{th}^{2}}{ }^{2}+\gamma_{\mathrm{th}}}{w \overline{\gamma_{1}}}-\frac{(K+1) w}{\overline{\gamma_{2}}}} I_{0}\left(2 \sqrt{\frac{K(K+1)\left(\gamma_{\mathrm{th}}+\mathrm{w}\right)}{\overline{\gamma_{2}}}}\right) d w$

Where $I_{0}($.$) is the zeroth order modified Bessel function of$ the first [10, Eq. (8.447.1)]. and by applying the integral result of [10, Eq. (3.471.9)] equation (10) can be solved. Finally the outage probability can be expressed in the form of

$P_{\text {out }}$

$=1-e^{-K-t \gamma_{\mathrm{th}}} \sum_{\mathrm{n}=0}^{\infty} \frac{\mathrm{K}^{\mathrm{n}}}{(n !)^{2}} \sum_{\mathrm{m}=0}^{\mathrm{n}}\left[\begin{array}{l}n \\ m\end{array}\right] \gamma_{\mathrm{th}}{ }^{n-m}\left(\frac{\mathrm{K}+1}{\overline{\gamma_{2}}}\right)^{\frac{2 \mathrm{n}-\mathrm{m}+1}{2}}$

$\left(\frac{\gamma_{\mathrm{th}}{ }^{2}+\mathrm{c} \gamma_{\mathrm{th}}}{\bar{\gamma}_{1}}\right)^{\frac{\mathrm{m}+1}{2}} \times K_{m+1}\left(2 \sqrt{\frac{(K+1)\left(\gamma_{\mathrm{th}}{ }^{2}+\mathrm{c} \gamma_{\mathrm{th}}\right)}{\bar{\gamma}_{1} \bar{\gamma}_{2}}}\right)$

where $\mathrm{K}_{\mathrm{n}+1}$ is modified Bessel function of the second kind $[10$, Eq. $(8.446)]$ and $t=\frac{\overline{\gamma_{2}}+(K+1) \overline{\gamma_{1}}}{\overline{\gamma_{1}} \overline{\gamma_{2}}}$

Several recent papers have approximated $\gamma_{e q}$ using an upper bound $\gamma_{b}$ given by

$\gamma_{b}=\min \left(\gamma_{1}, \gamma_{2}\right)$

Therefore, a closed form lower bound which become tight for high signal-to-noise ratios to equation (11) can be given by

$$
\begin{aligned}
P_{\text {out }} & =P_{\gamma}\left[\min \left(\gamma_{1}, \gamma_{2}\right)<\gamma_{t h}\right] \\
& =1-\mathrm{C}_{\gamma_{1}}\left(\gamma_{t h}\right) \mathrm{C}_{\gamma_{2}}\left(\gamma_{t h}\right) \\
P_{\text {out }}= & 1-e^{-\gamma_{t h} / \overline{\gamma_{1}}} Q\left(\sqrt{2 K}, \sqrt{\frac{2(1+K) \gamma_{t h}}{\overline{\gamma_{2}}}}\right)
\end{aligned}
$$

where $Q(.,$.$) is the first-order Marcum Q$-function [9].

\subsection{Average Bit Error Probability}

It is the one that is most revealing about the nature of the system behavior and the one most often illustrated in documents containing system performance evaluations; thus, it is of primary interest to have a method for its evaluation that reduces the degree of difficulty as much as possible. The primary reason for the difficulty in evaluating average bit error probability lies in the fact that the conditional (on the fading) bit error probability (BEP) is, in general, a nonlinear function of the instantaneous SNR, as the nature of the nonlinearity is a function of the modulation/detection scheme employed by the system[11].

Traditionally, the ABEP is computed by determining the PDF of $\gamma_{\mathrm{eq}}$ and then averaging the conditional BEP in AWGN, $\mathrm{P}_{\mathrm{b}}(\mathrm{e} / \gamma)$, over this PDF. Therefore, the average probability is defined as [9],

$\mathrm{P}_{\mathrm{b}}(\mathrm{e})=\int_{0}^{\infty} \mathrm{P}_{\mathrm{b}}\left(\frac{\mathrm{e}}{\gamma}\right) \mathrm{p}_{\gamma_{\mathrm{eq}}}(\gamma) \mathrm{d} \gamma$

Assume Conditional Probability is represented by Gaussian Q-function, i.e. $\mathrm{P}_{\mathrm{b}}(\mathrm{e} / \gamma)=\mathrm{Q}(\sqrt{\beta \gamma})$, where $\beta$ is constant and $\mathrm{Q}(\mathrm{x})$ is Gaussian Q-function. It is trivial to extend the following error performance derivations to well-known modulation schemes employed in communication systems such as $\operatorname{BPSK}(\beta=2)$ and $\operatorname{QPSK}(\beta=1)$.

In order to compute the ABEP of equation (14) by putting the Gaussian-Q function, then ABEP can be written as,

$\mathrm{P}_{\mathrm{b}}(\mathrm{e})=\frac{1}{\sqrt{2 \pi}} \int_{0}^{\infty} \mathrm{F}_{\gamma_{\text {eq }}}\left(\frac{\mathrm{t}^{2}}{\beta}\right) \mathrm{e}^{-\mathrm{t}^{2} / 2} \mathrm{dt}$

Now employing equation (13) in equation (15). The ABEP can be expressed as

$A B E P=P_{b}(e)=$

$\frac{1}{2}-\frac{1}{\sqrt{2 \pi}} \int_{0}^{\infty} e^{-\frac{2+\overline{\gamma_{1}}}{2 \overline{\gamma_{1}}} t^{2}} Q\left(\sqrt{2 K}, \sqrt{\frac{2(1+K)}{\overline{\gamma_{2}}}} t\right) d t$

By using [12, Eq. (6.621.3)], ABEP can be expressed in closed-form and is given by (17),

$\mathrm{ABEP}=\frac{1}{2}-\frac{v}{2}\left[1-2 Q\left(\sqrt{\frac{K}{2}}\left(1-\frac{1}{\sqrt{1+s}}\right), \sqrt{\frac{K}{2}}\left(1+\frac{1}{\sqrt{1+s}}\right)\right)+\right.$
$\left(1+\frac{1}{\sqrt{1+s}}\right) e^{\left.-K \frac{2+s}{2+2 s} I_{0}\left(\frac{K S}{2+2 s}\right)\right]}$

where $v=1 / \sqrt{1+\frac{2}{\beta \overline{\gamma_{1}}}} \quad \& \quad s=\frac{(1+K) \overline{\gamma_{1}}}{\left(1+\overline{\beta \gamma_{1}} / 2\right) \overline{\gamma_{2}}}$

\section{NUMERICAL RESULTS}

In this section, we present some numerical results to verify our analysis. Using MATLAB we plot the performance curves in terms of Outage Probability and Average Bit Error Probability, versus the Average SNR.

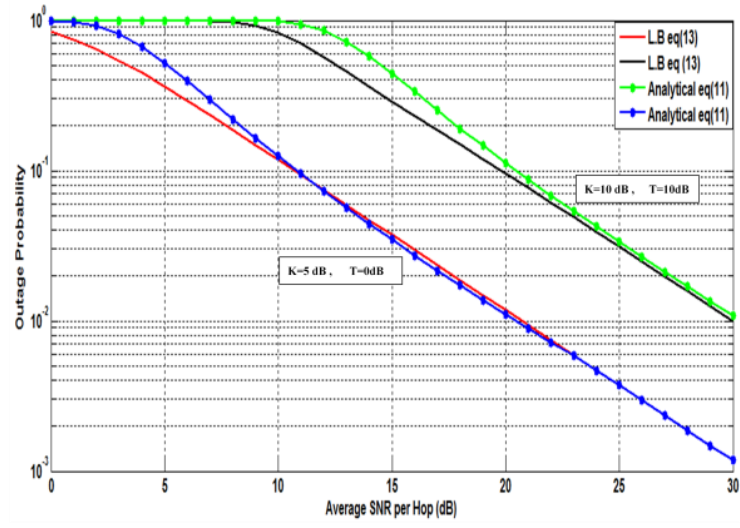

Fig. 2: Outage probability for various $K$ factor with

$$
\overline{\gamma_{1}}=\overline{\gamma_{2}}
$$

Fig. 2 shows the outage probability plot versus average SNR, for two different values of $\mathrm{K}$ Rician factors and threshold SNR(T). The analytical curves are from equation (11) using only 20 summations $(n=0, \ldots, 20)$, Furthermore, we see 
that the lower bound analytical curves from (13) converge at high SNR to the exact outage probability curves, as expected, the Outage Probability is decreased with the high value of SNR.

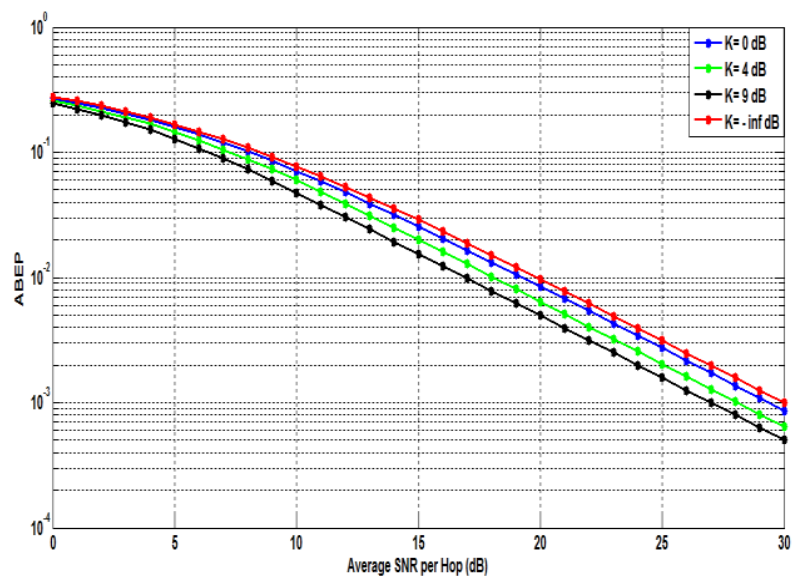

Fig. 3: ABEP versus average SNR (dB) for QPSK modulation scheme with $\overline{\gamma_{1}}=\overline{\gamma_{2}}$

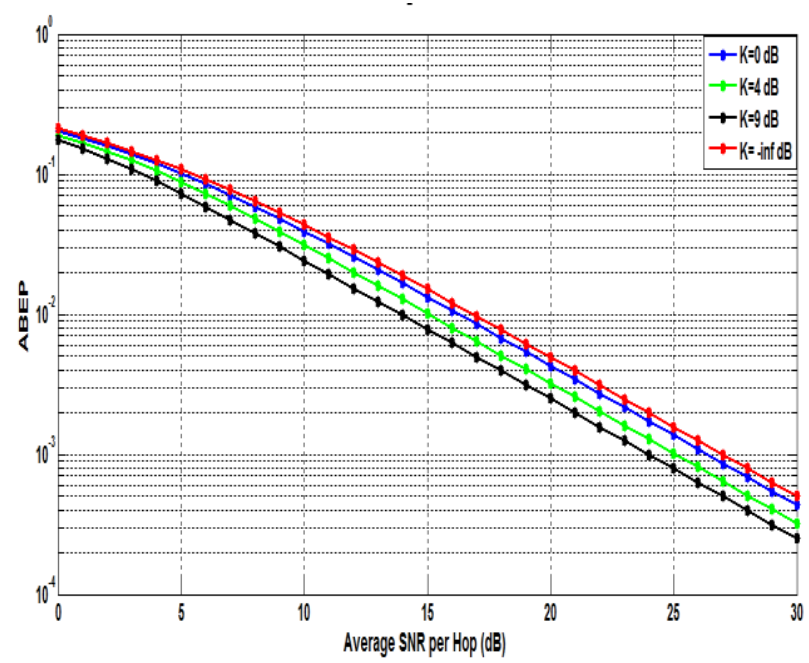

Fig. 4: ABEP versus average SNR (dB) for BPSK modulation scheme with $\overline{\gamma_{1}}=\overline{\gamma_{2}}$

Fig. 3 and Fig. 4 shows the ABEP plot versus Average SNR per Hop $\overline{\gamma_{1}}=\overline{\gamma_{2}}$, for various $\mathrm{K}$ Rician factors using QPSK and BPSK modulation schemes respectively. These results are obtained from the formula given in (17). We can derive similar conclusions that increasing the value of Rician fading parameter $\mathrm{K}$ lead to providing better performances of relay transmitting system, because ABEP then tends to smaller values. We observed from the plots, the system performance is better for BPSK modualtion scheme as compare to QPSK modulation scheme.

It can be seen from the table I that for $20 \mathrm{~dB}$ SNR, The average bit error probability is decreases with increase in the value of $\mathrm{K}$ and system gives better performance for BPSK modulation scheme.
Table 1. ABEP for QPSK and BPSK Modulation Scheme at Fixed SNR

\begin{tabular}{|c|c|c|c|}
\hline \multirow{2}{*}{$\begin{array}{l}\text { SNR } \\
\text { in } \\
(\mathrm{dB})\end{array}$} & \multirow{2}{*}{$\begin{array}{l}\text { Rician fading } \\
\text { parameter } \\
(\mathbf{K}) \text { in dB }\end{array}$} & \multicolumn{2}{|c|}{ Average Bit Error Probability } \\
\hline & & QPSK & BPSK \\
\hline 20 & $0 \mathrm{~dB}$ & 0.008496 & 0.004926 \\
\hline 20 & $9 \mathrm{~dB}$ & 0.004961 & 0.002494 \\
\hline
\end{tabular}

\section{CONCLUSION}

In this paper, we analyzed the system performance of Two Hop Relayed transmission in asymmetric Rayleigh and Rician fading environment. Asymmetric fading scenarios are used on those places where link having unequal signal strength. Closed form expressions of Outage probability and the Average bit error probability for QPSK and BPSK modulation schemes are derived to predict the performance of the proposed system. It is clear from the numerical results that for higher values of $\mathrm{K}$, better performance is achieved. Also the system gives better results for BPSK modulation scheme.

\section{REFERENCES}

[1] Lin Yang, Mazen O. Hasna and Mohamed-Slim Alouini, "Average Outage Duration of Multihop Communication Systems With Regenerative Relays," IEEE Transactions On Wireless Communications, Vol. 4, No. 4,pp.13661371, July 2005.

[2] Younsun Kim and Hui Liu, "Infrastructure Relay Transmission With Cooperative MIMO," IEEE Transactions On Vehicular Technology, Vol. 57, No. 4, pp. 2180-2188, 'July 2008.

[3] Mazen O. Hasna and Mohamed-Slim Alouini, "End-toEnd Performance of Transmission Systems With Relays Over Rayleigh-Fading Channels," IEEE Transactions On Wireless Communications, Vol. 2, No. 6, pp.1126-1131 ,November 2003.

[4] M. O. Hasna and M.-S. Alouini, "A performance study of dual-hop transmissions with fixed gain relays," IEEE Transactions on Wireless Communication., Vol. 3, pp. 1963-1968, Nov. 2004

[5] Mohammad Torabi, Wessam Ajib and David Haccoun, "Performance Analysis of Amplify-and-Forward Cooperative Networks with Relay Selection over Rayleigh Fading Channels," IEEE 69th Vechicular Technology Conference, 2009., pp.1-5,June 2009.

[6] Himal A. Suraweera, George K. Karagiannidis and Peter J. Smithand Peter J. Smith, "Performance Analysis of the Dual-Hop Asymmetric Fading Channel," IEEE Transactions On Wireless Communications, Vol. 8, No. 6,pp.2783-2788, June 2009.

[7] A. Adinoyi and H. Yanikomeroglu, "On the performance of cooperative wireless fixed relays in asymmetric channels," in Proc. IEEE GLOBECOM 2006, San Franscisco, CA, Dec. 2006, pp. 1-5.

[8] Kapil Gupta, P.K. Ghosh and Anup Dey, "End-to-End performance of a DPSK multi-input-multi-output relaying system in Rayleigh fading channels," Journal of Information Systems and Communication, Vol. 3, No. 1,pp.68-71, February 2012. 
[9] M. K. Simon and M.-S. Alouini, Digital Communication Over Fading Channels, $2^{\text {nd }}$ Edition, New York: A John Wiley and Sons Inc., 2005.

[10] I. S. Gradshteyn and I. M. Ryzhik, Table of Integrals, Series and Products. San Diego, CA: Academic Press, 2000 .
[11] Yang, P Fan, TQ Duong, X Lei, "Exact performance of two-way AF relaying in Nakagami-m fading envoirnment," IEEE Trans. Wireless. Communication. Vol.10, No.3,pp- 980-987, July 2012.

[12] A. H. Nuttall, "Some integrals involving the $Q$-function", Naval Underwater Systems Center (NUSC), Technical Report 4297, Apr. 1972. 